\title{
Positive Solutions for Schrödinger-Poisson Systems with Sign- Changing Potential and Critical Growth
}

\author{
Pengfei He $\mathbb{D}^{1,2}$ and Hongmin Suo $\mathbb{D}^{3}$ \\ ${ }^{1}$ School of Mathematics Science, Guizhou Normal University, Guiyang 550025, China \\ ${ }^{2}$ School of Mathematics and Statistics, Guizhou University of Finance and Economics, Guiyang 550025, China \\ ${ }^{3}$ School of Data Science and Information Engineering, Guizhou Minzu University, Guiyang 550025, China
}

Correspondence should be addressed to Pengfei He; gznchepengfei@163.com

Received 21 March 2020; Revised 16 May 2020; Accepted 10 June 2020; Published 2 July 2020

Academic Editor: Kasso Okoudjou

Copyright (c) 2020 Pengfei He and Hongmin Suo. This is an open access article distributed under the Creative Commons Attribution License, which permits unrestricted use, distribution, and reproduction in any medium, provided the original work is properly cited.

In this paper, we study the existence of positive solutions for Schrödinger-Poisson systems with sign-changing potential and critical growth. By using the analytic techniques and variational method, the existence and multiplicity of positive solutions are obtained.

\section{Introduction and Main Result}

In this paper, we consider the existence and multiplicity of positive solutions of the Schrödinger-Poisson systems with critical nonlinearity of the following form:

$$
\begin{cases}-\Delta u-\phi u=\lambda f_{\lambda}(x)|u|^{q-2} u+|u|^{4} u, & \text { in } \Omega, \\ -\Delta \phi=u^{2}, & \text { in } \Omega, \\ u=\phi=0, & \text { on } \partial \Omega,\end{cases}
$$

where $\Omega$ is a smooth bounded domain in $\mathbb{R}^{3}, 1<q<2, f_{\lambda}$ $=\lambda f^{+}+f^{-}, \lambda>0$, and $f^{ \pm}= \pm \max \{ \pm f, 0\}$. When $N=3,2^{*}$ $=(2 N /(N-2))=6$, the right hand side of the system of $(1)$ has the critical term $|u|^{4} u$. It is pointed out that system (1) is related to the following Schrödinger-Poisson system:

$$
\begin{cases}-\Delta u+V(x) u+k(x) \phi|u|^{q-2} u=f(x, u), & \text { in } \mathbb{R}^{3}, \\ -\Delta \phi=k(x)|u|^{q}, & \text { in } \mathbb{R}^{3},\end{cases}
$$

where the potentials $V(x), k(x)$ satisfy some mild conditions. The existence, uniqueness, and multiplicity of positive solutions of systems like (2) have been extensively studied in the last few decades, such as [1-14]. Furthermore, the Schrödinger-Poisson systems involving critical growth have been attracted many researchers, e.g., [15-24]. But there is little literature for liking the system (1) that considers the critical growth on bounded domains, such as [15-17].

In [24], the authors considered a Schrödinger-Poisson system with concave-convex nonlinearities of the form

$$
\begin{cases}-\Delta u+\lambda \phi u=\lambda u^{q-1}+u^{5}, & \text { in } \Omega, \\ -\Delta \phi=|u|^{2}, & \text { in } \Omega, \\ u=\phi=0, & \text { on } \partial \Omega,\end{cases}
$$

where $\Omega$ is a smooth bounded domain in $\mathbb{R}^{3}, 1<q<2$ and $\lambda>0$. They obtained two positive solutions when $\lambda$ is enough small by using the concentration-compactness principle.

Recently, in [25], the authors considered a SchrödingerNewton system with critical nonlinearities of the form

$$
\begin{cases}-\Delta u=f_{\lambda}(x)|u|^{q-2} u+\phi|u|^{3} u, & \text { in } \Omega, \\ -\Delta \phi=|u|^{5}, & \text { in } \Omega, \\ u=\phi=0, & \text { on } \partial \Omega,\end{cases}
$$


where $\Omega$ is a smooth bounded domain in $\mathbb{R}^{3}, 1<q<2, f_{\lambda}$ $=\lambda f^{+}+f^{-}, \lambda>0$, and $f^{ \pm}= \pm \max \{ \pm f, 0\}$. The authors studied the existence and multiplicity of positive solutions by the variational method and analytic techniques.

Motivated by results found in the above mentioned papers, in the present paper, we intend to study the existence of multiple positive solutions for system (1). Since system (1) contains a critical term and a nonlocal term, the critical value level becomes very difficult to estimate. The main objective of this paper is to look for new estimates and establish two positive solutions by analytic techniques.

The main result is as follows:

Theorem 1. Assume that $1<q<2$ and $f_{\lambda} \in L^{\infty}(\Omega)$ is a possibly sign-changing function. Then, there exists $\lambda^{*}>0$ such that for any $\lambda \in\left(0, \lambda^{*}\right)$, system (1) has at least two different positive solutions. Additionally, system (1) admits a positive ground state solution.

Throughout this paper, we use the following notations:

(i) The space $H_{0}^{1}(\Omega)$ is equipped with the norm $\|u\|^{2}=$ $\int_{\Omega}|\nabla u|^{2} d x$. The norm in $L^{p}(\Omega)$ is denoted by $|\cdot|_{p}$

(ii) $C, C_{1}, C_{2}, \cdots$ denote various positive constants, which may vary from line to line

(iii) We denote by $B(0, r)$ (respectively, $\partial B(0, r)$ ) the closed ball (respectively, the sphere) of center zero and radius $r$, i.e., $B(0, r)=\left\{u \in H_{0}^{1}(\Omega):\|u\| \leq r\right\}$, $\partial B(0, r)=\left\{u \in H_{0}^{l}(\Omega):\|u\|=r\right\}$

(iv) Let $S$ be the best Sobolev embedding constant for the embedding $H_{0}^{1}(\Omega) \mapsto L^{6}(\Omega)$, namely,

$$
S=\inf _{u \in H_{0}^{1}(\Omega) \backslash\{0\}} \frac{\int_{\Omega}|\nabla u|^{2} d x}{\left(\int_{\Omega}|u|^{6} d x\right)^{1 / 3}}
$$

\section{Existence of a First Positive Solution of System (1)}

For any given $u \in H_{0}^{1}(\Omega)$, by the Lax-Milgram theorem, the Dirichlet boundary problem $-\Delta \phi=u^{2}$ in $\Omega$ has a unique solution $\phi_{u} \in H_{0}^{1}(\Omega)$. Substitute $\phi_{u}$ in the first equation of system (1), system (1) transformed into the following variable equations:

$$
\begin{cases}-\Delta u-\phi u=\lambda f_{\lambda}(x)|u|^{q-2} u+|u|^{4} u, & \text { in } \Omega, \\ -\Delta \phi=u^{2}, & \text { on } \partial \Omega .\end{cases}
$$

The formal energy functional corresponding to the system (6) is the following:

$I_{\lambda}(u)=\frac{1}{2}\|u\|^{2}-\frac{1}{4} \int_{\Omega} \phi_{u}|u|^{2} d x-\frac{1}{q} \int_{\Omega} f_{\lambda}(x)|u|^{q} d x-\frac{1}{6} \int_{\Omega}|u|^{6} d x$.
If a function $u \in H_{0}^{1}(\Omega)$ satisfies

$$
\begin{aligned}
& \int_{\Omega}(\nabla u, \nabla v) d x-\int_{\Omega} f_{\lambda}(x) u^{q-1} v d x-\int_{\Omega} \phi_{u} u v d x-\int_{\Omega} u^{5} v d x \\
& \quad=0, \quad \forall v \in H_{0}^{1}(\Omega),
\end{aligned}
$$

where $v \in H_{0}^{1}(\Omega)$, we can prove that $(u, \phi) \in H_{0}^{1}(\Omega) \times H_{0}^{1}(\Omega)$ is a solution of system (1) if and only if $\phi=\phi_{u}$, and $u \in H_{0}^{1}(\Omega)$ is called a weak solution of (6).

To prove our main theorem 1 , some preliminary results are needed, let us first collect some properties.

Lemma 2 (see [17]). For each $u \in H_{0}^{1}(\Omega)$, there exists a unique solution $\phi_{u} \in H_{0}^{1}(\Omega)$ of

$$
\begin{cases}-\Delta \phi=|u|^{2}, & \text { in } \Omega, \\ \phi=0, & \text { on } \Omega\end{cases}
$$

Moreover,

(1) $\phi_{u} \geq 0$ for $x \in \Omega$

(2) For each $t \neq 0, \phi_{t u}=t^{2} \phi_{u}$

(3) $\int_{\Omega} \phi_{u}|u|^{2} d x=\int_{\Omega}\left|\nabla \phi_{u}\right|^{2} d x \leq S^{-3}\|u\|^{4}$

(4) Suppose that $u_{n} \rightarrow u$ in $H_{0}^{1}(\Omega)$, then

$$
\int_{\Omega} \phi_{u_{n}}\left|u_{n}\right|^{2} d x-\int_{\Omega} \phi_{u_{n}-u}\left|u_{n}-u\right|^{2} d x=\int_{\Omega} \phi_{u}|u|^{2} d x+o(1)
$$

Lemma 3 (see [18]). Let $r, s>1, \psi \in L^{s}(\Omega)$, and $\psi^{+}=\max$ $(\psi, 0) \neq 0$, then there exists $\varphi_{0} \in C_{0}^{\infty}(\Omega)$ such that $\int_{\Omega} \psi$ $\left|\varphi_{0}\right|^{r} d x>0$.

Lemma 4. There exist $\alpha, \rho, \Lambda_{0}>0$, such that every $\lambda \in\left(0, \Lambda_{0}\right)$, we have

$$
\begin{array}{ll}
I_{\lambda}(u)>0 & \text { for } u \in \partial B(0, \rho), \\
I_{\lambda}(u)<0 & \text { for } u \in B(0, \rho) .
\end{array}
$$

Proof. According to the Sobolev and Hölder inequalities and Lemma 2, we have

$$
\begin{aligned}
I_{\lambda}(u)= & \frac{1}{2}\|u\|^{2}-\frac{1}{4} \int_{\Omega} \phi_{u}|u|^{2} d x-\frac{1}{q} \int_{\Omega} f_{\lambda}(x)|u|^{q} d x-\frac{1}{6} \int_{\Omega}|u|^{6} d \\
\geq & \frac{1}{2}\|u\|^{2}-\frac{1}{4} S^{-3}\|u\|^{4}-\frac{\lambda}{q}|\Omega|^{(6-q) / 6} S^{-q / 2}\left|f^{+}\right|_{\infty}\|u\|^{q} \\
& -\frac{1}{6} S^{-3}\|u\|^{6}=\|u\|^{q}\left[\frac{1}{2}\|u\|^{2-q}-\frac{1}{4} S^{-3}\|u\|^{4-q}\right. \\
& \left.-\frac{1}{6} S^{-3}\|u\|^{6-q}-\frac{\lambda}{q}|\Omega|^{(6-q) / 6} S^{-q / 2}\left|f^{+}\right|_{\infty}\right] .
\end{aligned}
$$


Setting $g(t)=(1 / 2) t^{2-q}-(1 / 4) S^{-3} t^{4-q}-(1 / 6) S^{-3} t^{6-q}$ for $t>0$, there exists a positive constant

$\rho=\left[\frac{(1 / 2)(4-q) S^{-3}-\sqrt{(1 / 4)(4-q)^{2} S^{-1}+(4 / 3)(6-q)(2-q) S^{-3}}}{(2 / 3)(6-q) S^{-3}}\right]^{1 / 2}>0$,

such that $\max _{t>0} g(t)=g(\rho)>0$. Letting $\Lambda_{0}=\left(q S^{9 / 2} / 2\left|f^{+}\right|_{\infty}\right.$ $\left.|\Omega|^{(6-q) / 6}\right) g(\rho)$. Consequently, it follows that $\left.I_{\lambda}\right|_{\|u\|=\rho} \geq \alpha \triangleq$ $(g(\rho) / 6) \rho^{q}$ for any $\lambda \in\left(0, \Lambda_{0}\right)$.

Additionally, by Lemma 3 , there exists $\varphi_{0} \in C_{0}^{\infty}(\Omega) \subset$ $H_{0}^{1}(\Omega)$ such that

$$
\lim _{t \rightarrow 0^{+}} \frac{I_{\lambda}(t u)}{t^{q}}=\frac{1}{q} \int_{\Omega} f_{\lambda}(x)\left|\varphi_{0}\right|^{q} d x .
$$

For $u$ small enough, we have $I_{\lambda}(u)<0$. The lemma is proved.

We recall that for $c \in \mathbb{R}$, a sequence $\left\{u_{n}\right\} \subset H_{0}^{1}(\Omega)$ is called a $(P S)_{c}$ sequence for $I$ if $I\left(u_{n}\right)=c+o(1)$ and $I^{\prime}\left(u_{n}\right)$ $=o(1)$ strongly in the dual space of $H_{0}^{1}(\Omega)$ as $n \rightarrow \infty$. That is to say, $I$ satisfies the $(P S)_{c}$ condition in $H_{0}^{1}(\Omega)$ if every $(P S)_{c}$ sequence in $H_{0}^{1}(\Omega)$ for $I$ contains a convergent subsequence.

Lemma 5. If $\left\{u_{n}\right\} \subset H_{0}^{1}(\Omega)$ is a $(P S)_{c}$ sequence for $I_{\lambda}$ with $c$ $<(1 / 3) S^{3 / 2}-D \lambda^{2 /(2-q)} \quad$ where $D=D\left(|\Omega|, S, q,\left|f^{+}\right|_{\infty}\right)>0$. Then, there exists $u \in H_{0}^{1}(\Omega)$ such that $u_{n} \rightarrow u_{*}$ in $H_{0}^{1}(\Omega)$ and $\int_{\Omega}\left|u_{n}\right|^{6} d x \rightarrow \int_{\Omega}|u|^{6} d x$.

Proof. Let $\{u\} \in H_{0}^{1}(\Omega)$ be such that

$$
I_{\lambda}\left(u_{n}\right) \rightarrow c, I_{\lambda}^{\prime}\left(u_{n}\right) \rightarrow 0 .
$$

We claim that $\left\{u_{n}\right\}$ is bounded in $H_{0}^{1}(\Omega)$. Indeed, by (15), we have that

$$
\begin{aligned}
1+c+o(1)\left\|u_{n}\right\| & \geq I_{\lambda}\left(u_{n}\right)-\frac{1}{4}\left\langle I_{\lambda}^{\prime}\left(u_{n}\right), u_{n}\right\rangle \\
& \geq \frac{1}{4}\left\|u_{n}\right\|^{2}-\left(\frac{1}{q}-\frac{1}{4}\right) \int_{\Omega} f_{\lambda}(x)\left|u_{n}\right|^{q} d x \\
& \geq \frac{1}{4}\left\|u_{n}\right\|^{2}-\lambda\left(\frac{1}{q}-\frac{1}{4}\right)|\Omega|^{(6-q) / 6} S^{-q / 2}\left|f^{+}\right|_{\infty}\left\|u_{n}\right\|^{q},
\end{aligned}
$$

which implies that $\left\{u_{n}\right\}$ is bounded in $H_{0}^{1}(\Omega)$. Thereby, there exists a subsequence, still denoted by $\left\{u_{n}\right\}$, and a function $u_{*} \in H_{0}^{1}(\Omega)$, such that

$$
\left\{\begin{array}{l}
u_{n} \rightarrow u, \text { weakly in } H_{0}^{1}(\Omega), \\
u_{n} \rightarrow u, \text { strongly in } L^{s}(\Omega)(1 \leq s<6), \\
u_{n}(x) \rightarrow u(x), \text { a.e.in } \Omega,
\end{array}\right.
$$

as $n \rightarrow \infty$. Set $w_{n}=u_{n}-u$, then $\left\|w_{n}\right\| \rightarrow 0$. Otherwise, there exists a subsequence (still denoted by $w_{n}$ ) such that

$$
\lim _{n \rightarrow \infty}\left\|w_{n}\right\|=l>0 .
$$

By (15), letting $n \rightarrow \infty$, for every $\varphi \in H_{0}^{1}(\Omega)$, it follows

$\int_{\Omega}(\nabla u, \nabla \varphi) d x-\int_{\Omega} f_{\lambda}(x)|u|^{q-2} u \varphi d x-\int_{\Omega} \phi_{u} u \varphi d x-\int_{\Omega}|u|^{4} u \varphi d x=0$. have

In particular, taking the test function $\varphi=u$ in (19), we

$$
\|u\|^{2}-\int_{\Omega} f_{\lambda}(x)|u|^{q} d x-\int_{\Omega} \phi_{u}|u|^{2} d x-\int_{\Omega}|u|^{6} d x=0 .
$$

By Brézis-Lieb (see [26]) and Lemma 2, one has

$$
\left\{\begin{array}{l}
\left\|u_{n}\right\|^{2}=\left\|w_{n}\right\|^{2}+\|u\|^{2}+o(1) \\
\int_{\Omega}\left|u_{n}\right|^{6} d x=\int_{\Omega}\left|w_{n}\right|^{6} d x+\int_{\Omega}|u|^{6} d x+o(1) .
\end{array}\right.
$$

Therefore, according to $\left\langle I_{\lambda}^{\prime}\left(u_{n}\right), u_{n}\right\rangle \rightarrow 0$ as $n \rightarrow \infty$, we have

$$
\begin{aligned}
\|u\|^{2} & +\left\|w_{n}\right\|^{2}+\int_{\Omega} \phi_{u}|u|^{2} d x-\int_{\Omega}|u|^{6} d x \\
& -\int_{\Omega}\left|w_{n}\right|^{6} d x-\int_{\Omega} f_{\lambda}(x)|u|^{q} d x=o(1) .
\end{aligned}
$$

It follows from (20) and (22) implies that

$$
\left\|w_{n}\right\|^{2}-\int_{\Omega}\left|w_{n}\right|^{6} d x=o(1) .
$$

Note that $\int_{\Omega}\left|w_{n}\right|^{6} d x \leq S^{-3}\left\|w_{n}\right\|^{6}$, we deduce that

$$
l^{2} \geq S^{3 / 2} \text {. }
$$

On the one hand, by using the Young inequality and (20), there holds

$$
\begin{aligned}
I_{\lambda}\left(u_{*}\right)= & \frac{1}{2}\|u\|^{2}-\frac{1}{4} \int_{\Omega} \phi_{u}\left|u_{*}\right|^{2} d x-\frac{1}{6} \int_{\Omega}|u|^{6} d x \\
& -\frac{1}{q} \int_{\Omega} f_{\lambda}(x)|u|^{q} d x \geq \frac{1}{4}\|u\|^{2}-\left[\frac{1}{q}-\frac{1}{4}\right] \int_{\Omega} f_{\lambda}(x)|u|^{q} d x \\
\geq & \frac{1}{4}\|u\|^{2}-\lambda\left[\frac{1}{q}-\frac{1}{4}\right]|\Omega|^{(6-q) / 6} S^{-q / 2}\left|f^{+}\right|_{\infty}\|u\|^{q} \\
\geq & -D \lambda^{2 /(2-q)}
\end{aligned}
$$

where $D=D\left(|\Omega|, S, q,\left|f^{+}\right|_{\infty}\right)>0$ is a constant. 
On the other hand, note that $c<(1 / 3) S^{3 / 2}-D \lambda^{2 /(2-q)}$, by (23), one has

$$
\begin{aligned}
I_{\lambda}\left(u_{*}\right) & =I_{\lambda}\left(u_{n}\right)-\frac{1}{2}\left\|w_{n}\right\|^{2}-\frac{1}{6} \int_{\Omega}\left|w_{n}\right|^{6} d x+o(1) \\
& =I_{\lambda}\left(u_{n}\right)-\frac{1}{3}\left\|w_{n}\right\|^{2}+o(1) \\
& \leq c-\frac{1}{3} l^{2}<\frac{1}{3} S^{3 / 2}-D \lambda^{2 /(2-q)}-\frac{1}{3} S^{3 / 2} \\
& =-D \lambda^{2 /(2-q)} .
\end{aligned}
$$

From the above analysis, this is a contradiction. Therefore, $l=0$, it implies that $u_{n} \rightarrow u$ in $H_{0}^{1}(\Omega)$, and

$$
\begin{aligned}
0 & \leq \int_{\Omega}\left|u_{n}\right|^{6} d x-\int_{\Omega}|u|^{6} d x=\int_{\Omega}\left|w_{n}\right|^{6} d x+o(1) \\
& \leq S^{-3}\left\|w_{n}\right\|^{6}+o(1) \longrightarrow 0 .
\end{aligned}
$$

So $\int_{\Omega}\left|u_{n}\right|^{6} d x \rightarrow \int_{\Omega}|u|^{6} d x$ as $n \rightarrow \infty$. The lemma is proved.

Theorem 6. Assume $1<q<2$ and $f_{\lambda} \in L^{\infty}(\Omega)$. Then, system (1) has a positive solution $\left(u_{*}, \phi_{u_{*}}\right) \in H_{0}^{1}(\Omega) \times H_{0}^{1}(\Omega)$ which satisfies $I_{\lambda}\left(u_{*}\right)<0$.

Proof. From Lemma 4 and applying Ekeland's variational principle in $\overline{B_{\rho}(0)}$, there exists a minimizing sequence $\left(u_{n}\right)$ $\subset \overline{B_{\rho}(0)}$ such that

$$
\begin{aligned}
I_{\lambda}\left(u_{n}\right) & \leq \inf _{u_{n} \in \overline{B_{\rho}(0)}} I_{\lambda}(u)+\frac{1}{q}, I_{\lambda}(v) \\
& \geq I_{\lambda}\left(u_{n}\right)-\frac{1}{n}\left\|v-u_{n}\right\|, \quad \forall u_{n} \in \overline{B_{\rho}(0)} .
\end{aligned}
$$

Therefore,

$$
\begin{gathered}
I_{\lambda}^{\prime}\left(u_{n}\right) \rightarrow 0, \\
I_{\lambda}\left(u_{n}\right)<c .
\end{gathered}
$$

Since $\left\{u_{n}\right\}$ is bounded in $H_{0}^{1}(\Omega)$, there exist $u_{*} \in \overline{B_{\rho}(0)}$ and a subsequence still denoted by $\left\{u_{n}\right\}$ such that $u_{n} \rightarrow u_{*}$ in $H_{0}^{1}(\Omega)$ as $n \rightarrow \infty$.

Note that $I_{\lambda}\left(u_{n}\right)=I_{\lambda}\left(\left|u_{n}\right|\right)$ and Lemma 5, we can obtain $u_{n} \rightarrow u_{*}$ in $H_{0}^{1}(\Omega)$ and $u_{*} \geq 0$ and $u_{*} \neq 0$. Since $u_{*} \in H_{0}^{1}(\Omega)$, the embedding theorem means $u_{*} \in L^{6}(\Omega)$. Note that $f_{\lambda} \in$ $L^{\infty}(\Omega)$, by the regularity of weak solutions, one has $u_{*} \epsilon$ $W^{2,6 / q}(\Omega)$. Thereby, according to the classical bootstrap argument, we have $u_{*} \in W^{1, \alpha}$ for $r \in[1,+\infty)$. By the embedding theorem again, it holds that $u_{*} \in C^{1, \alpha}(\Omega)$ for $0<\alpha<1$. Since $\phi_{u_{*}} \geq 0$, we have

$$
-\Delta u_{*}=u_{*}^{5}+f_{\lambda}(x) u_{*}^{q-1}+\phi_{u *} u_{*} \geq-f^{-}(x) \mid u_{*}^{q-1} \geq-C u_{*}
$$

for some $C>0$. Thereby, by the strong maximum principle, we obtain $u_{*}>0$ in $\Omega$, then $\left(u_{*}, \phi_{u_{*}}\right)$ is a positive solution of system (1) with $I_{\lambda}\left(u_{*}\right)<0$ (by (11)). The theorem is proved.

For $u \in H_{0}^{1}(\Omega)$, we have

$$
\begin{aligned}
I_{\lambda}(u)= & \frac{1}{2}\|u\|^{2}-\frac{1}{4} \int_{\Omega} \phi_{u}|u|^{2} d x-\frac{1}{6} \int_{\Omega}|u|^{6} d x \\
& -\frac{1}{q} \int_{\Omega} f_{\lambda}(x)|u|^{q} d x \leq \frac{1}{2}\|u\|^{2}-\frac{1}{6} \int_{\Omega}|u|^{6} d x \\
& -\frac{1}{q} \int_{\Omega} f_{\lambda}(x)|u|^{q} d x .
\end{aligned}
$$

Now, if we consider the following problem:

$$
\begin{cases}-\Delta u=f_{\lambda}(x)|u|^{q-2} u+|u|^{4} u, & \text { in } \Omega, \\ u>0, & \text { in } \Omega, \\ u=0, & \text { on } \partial \Omega .\end{cases}
$$

Then, we find the weak solutions of problem (32) correspond to the critical points of the functional $J_{\lambda}$, where

$$
J_{\lambda}(u)=\frac{1}{2}\|u\|^{2}-\frac{1}{q} \int_{\Omega} f_{\lambda}(x)|u|^{q} d x-\frac{1}{6} \int_{\Omega}|u|^{6} d x .
$$

Obviously, one has

$$
I_{\lambda}(u) \leq J_{\lambda}(u), \quad \text { for } u \in H_{0}^{1}(\Omega) .
$$

Remark 7. Problem (32) has a positive local minimizer solution $v_{0} \in B_{\rho}$ with $J_{\lambda}\left(v_{0}\right)<0$ for each $\lambda \in\left(0, \Lambda_{0}\right)$ where $\rho, \Lambda_{0}$ are as in Lemma 4.

Indeed, by Lemma 4, we have

$$
\frac{1}{2} \rho^{2-q}-\frac{1}{4} S^{-3} \rho^{4-q}-\frac{\lambda}{q}|\Omega|^{(6-q) / 6} S^{-q / 2}\left|f^{+}\right|_{\infty}>\frac{1}{6} S^{-3} \rho^{6-q} \text {. }
$$

Therefore, when $\|u\|=\rho$, there holds

$$
\begin{aligned}
J_{\lambda}(u) & =\frac{1}{2}\|u\|^{2}-\frac{1}{q} \int_{\Omega} f_{\lambda}(x)|u|^{q} d x-\frac{1}{6} \int_{\Omega}|u|^{6} d x \\
& \geq \frac{1}{2}\|u\|^{2}-\frac{S^{-3}}{6}\|u\|^{6}-\frac{\lambda|\Omega|^{(6-q) / 6}}{q} S^{-q / 2}\left|f^{+}\right|_{\infty}\|u\|^{q} \\
& =\|u\|^{q}\left(\frac{1}{2}\|u\|^{2-q}-\frac{S^{-3}}{6}\|u\|^{6-q}-\frac{\lambda|\Omega|^{(6-q) / 6}}{q} S^{-q / 2}\left|f^{+}\right|_{\infty}\right) \\
& \geq \frac{1}{6} S^{-3} \rho^{6-q} \rho^{q}=\frac{1}{6} S^{-3} \rho^{6} .
\end{aligned}
$$

Similar to Lemma 4, we have

$$
\lim _{t \rightarrow 0^{+}} \frac{J_{\lambda}(t u)}{t^{q}}=\frac{1}{q} \int_{\Omega} f_{\lambda}(x)\left|\varphi_{0}\right|^{q} d x .
$$


So, we get $J_{\lambda}(u)<0$. Similar to Theorem 6 , problem (32) has a positive local minimizer solution $v_{0} \in B_{\rho}$ with $J_{\lambda}\left(v_{0}\right)$ $<0$ for each $\lambda \in\left(0, \Lambda_{0}\right)$.

\section{Existence of a Second Positive Solution of System (1)}

As usual, we consider the following function:

$$
v_{\varepsilon}=\frac{\left[3 \varepsilon^{2}\right]^{1 / 4}}{\left(\varepsilon^{2}+|x|^{2}\right)^{1 / 2}},
$$

where $\varepsilon$ is a positive constant. Moreover, we know that $v_{\varepsilon}$ is a positive solution of problem $-\Delta u=|u|^{4} u$ in $\mathbb{R}^{3}$ and $\int_{\Omega}$ $\left|\nabla v_{\varepsilon}\right|^{2} d x=\int_{\Omega}\left|v_{\varepsilon}\right|^{6} d x=S^{3 / 2}$. Let $\zeta$ be a smooth cut-off function $\zeta \in C_{0}^{\infty}(\Omega)$ such that $0 \leq \zeta(x) \leq 1$ in $\Omega, \zeta(x)=1$ near $x$ $=0$ and it is radially symmetric. Set $u_{\varepsilon}(x)=v_{\varepsilon}(x) \zeta(x)$ for $x$ $\in \Omega$. Then, we have

Lemma 8. Assume $1<q<2$, there holds

$$
\sup _{t \geq 0} I_{\lambda}\left(v_{0}+t u_{\varepsilon}\right)<\frac{1}{3} S^{3 / 2}-D \lambda^{2 /(2-q)} .
$$

Proof. According to the definition and from [27], there hold

$$
\begin{gathered}
\left\|u_{\varepsilon}\right\|^{2} \leq S^{3 / 2}+O(\varepsilon), \\
\int_{\Omega} u_{\varepsilon}^{6} d x \geq S^{3 / 2}+O(\varepsilon) .
\end{gathered}
$$

It is well known that the following inequality

$$
(a+b)^{6} \geq a^{6}+b^{6}+6 a^{5} b+6 a b^{5}
$$

holds for $a, b \geq 0$. Since $v_{0} \in B_{\rho_{1}}$ is a positive solution of the problem (32), and $J_{\lambda}<0$ for each $\lambda \in\left(0, \Lambda_{1}\right)$. Obviously, there exist two constants $n, M>0$ such that $m \leq v_{0}(x) \leq M$, using (41), there holds

$$
\begin{aligned}
J_{\lambda}\left(v_{0}+t u_{\varepsilon}\right)= & \frac{1}{2}\left(\left\|v_{0}\right\|^{2}+t^{2}\left\|u_{\varepsilon}\right\|^{2}\right)+\int_{\Omega}\left(\nabla v_{0}, \nabla t u_{\varepsilon}\right) d x \\
& -\frac{1}{6} \int_{\Omega}\left|v_{0}+t u_{\varepsilon}\right|^{6} d x-\frac{1}{q} \int_{\Omega} f_{\lambda}(x)\left|v_{0}+t u_{\lambda}\right|^{q} d x \\
\leq & J_{\lambda}\left(v_{0}\right)+\frac{t^{2}}{2}\left\|u_{\varepsilon}\right\|^{2}-\frac{1}{6} \int_{\Omega}\left[\left|v_{0}+t u_{\varepsilon}\right|^{6}-v_{0}^{6}-6 v_{0}^{5} t u_{\varepsilon}\right] d x \\
& +\frac{1}{q} \int_{\Omega}\left|f^{-}\right|_{\infty}\left\{\int_{0}^{t u_{\varepsilon}}\left[\left(v_{0}+\eta\right)^{q-1}-v_{0}^{q-1}\right] d \eta\right\} d x \\
\leq & \frac{t^{2}}{2}\left\|u_{\varepsilon}\right\|^{2}-\frac{1}{6} \int_{\Omega}\left[\left|t u_{\varepsilon}\right|^{6}+6 v_{0}\left(t u_{\varepsilon}\right)^{5}\right] d x \\
& +\frac{1}{q} \int_{\Omega}\left(\left|f^{-}\right|_{\infty} \int_{0}^{t u_{\varepsilon}} \eta^{q-1}\right) d x \\
\leq & \frac{t^{2}}{2}\left\|u_{\varepsilon}\right\|^{2}-\frac{t^{2}}{6} \int_{\Omega} u_{\varepsilon}^{6} d x-C_{1} t^{5} \int_{\Omega} u_{\varepsilon}^{5} d x \\
& +C_{2} t^{q} \int_{\Omega} u_{\varepsilon}^{q} d x,
\end{aligned}
$$

where $C_{1}, C_{2}>0$ (independent of $\varepsilon, \lambda$ ). Let

$$
h(t)=\frac{t^{2}}{2}\left\|u_{\varepsilon}\right\|^{2}-\frac{t^{2}}{6} \int_{\Omega} u_{\varepsilon}^{6} d x-C_{1} t^{5} \int_{\Omega} u_{\varepsilon}^{5} d x+C_{2} t^{q} \int_{\Omega} u_{\varepsilon}^{q} d x .
$$

Since $\lim _{t \rightarrow 0} h(t)=0, \lim _{t \rightarrow \infty} h(t)=-\infty$, it is similar to [25] that we prove that there exist $t_{\varepsilon}$ and positive constants $t_{1}, t_{2}$ independent of $\varepsilon, \lambda$, such that

$$
\begin{gathered}
0<t_{0} \leq t_{\varepsilon} \leq t_{2}<+\infty \\
\sup _{t \geq 0} h(t) \leq \frac{1}{3} S^{3 / 2}+C_{3} \varepsilon-C_{1} t_{1}^{5} \int_{\Omega} u_{\varepsilon}^{5} d x+C_{2} t_{2}^{q} \int_{\Omega} u_{\varepsilon}^{q} d x .
\end{gathered}
$$

Note that $\int_{\Omega} u_{\varepsilon}^{5} d x=C \varepsilon^{1 / 2}+O\left(\varepsilon^{1 / 2}\right), \quad \int_{\Omega} u_{\varepsilon}^{q} d x=C \varepsilon^{q / 2}$. Then,

$$
\begin{aligned}
\sup _{t \geq 0} h(t) & \leq \frac{1}{3} S^{3 / 2}+C_{3} \varepsilon-C_{4} \varepsilon^{1 / 2}+C_{5} \varepsilon^{q / 2} \\
& \leq \frac{1}{3} S^{3 / 2}+\left(C_{3}+C_{5}\right) \varepsilon^{q / 2}-C_{4} \varepsilon^{1 / 2} .
\end{aligned}
$$

where $C_{3}, C_{4}, C_{5}>0$ (independent of $\left.\varepsilon, \lambda\right)$. Let $\varepsilon=\lambda^{4 /(q(2-q))}$, $0<\lambda<\Lambda_{1}:=\left(C_{4} /\left(C_{3}+C_{5}+D\right)\right)^{2 /(2(q-1))}$, there holds

$$
\begin{aligned}
\left(C_{3}+C_{5}\right) \varepsilon^{q / 2}-C_{4} \varepsilon^{1 / 2} & =\left(C_{3}+C_{5}\right) \lambda^{2 /(2-q)}-C_{4} \lambda^{2 /(q(2-q))} \\
& =\lambda^{2 /(2-q)}\left(C_{3}+C_{5}-C_{4} \lambda^{2 /(q(2-q))}\right) \\
& <-D \lambda^{2 /(2-q)} .
\end{aligned}
$$

Recall that

$$
I_{\lambda}(u) \leq J_{\lambda}(u)
$$

for all $u \in H_{0}^{1}(\Omega)$. Therefore, from the above analysis, we conclude that (39) holds true when $\lambda<\Lambda_{1}$. The lemma is proved.

Theorem 9. Assume $1<q<2$ and $f_{\lambda} \in L^{\infty}(\Omega)$. Then, the system (1) admits a positive solution $\left(u_{*}, \phi_{u_{*}}\right) \in H_{0}^{1}(\Omega) \times H_{0}^{1}(\Omega)$ with $I_{\lambda}\left(u_{*}\right)>0$.

Proof. Let $\lambda_{*}=\min \left\{\Lambda_{0}, \Lambda_{1}, 1\right\}$, then Lemma 4 and Theorem 6 and Lemma 8 hold for $0<\lambda<\lambda_{*}$. By Remark 7, we can choose a large enough $T_{0}>0$ such that $I_{\lambda}\left(v_{0}+T_{0} u_{\varepsilon}\right)<0$. Consequently, $I_{\lambda}$ satisfies the mountain geometry structure. Applying the mountain-pass lemma [28], there exists a sequence $\left\{u_{n}\right\} \subset H_{0}^{1}(\Omega)$ such that

$$
I_{\lambda}^{\prime}\left(u_{n}\right) \rightarrow 0 \text { and } I_{\lambda}\left(u_{n}\right) \rightarrow c>0 \text { as } n \rightarrow \infty
$$


where

$$
\begin{gathered}
c=\inf _{\gamma \in \Gamma} \max _{t \in[0,1]} I_{\lambda}(\gamma(t)), \\
\Gamma=\left\{\gamma \in C\left([0,1], H_{0}^{1}(\Omega)\right): \gamma(0)=v_{0}, \gamma(1)=v_{0}+T_{0} u_{\varepsilon}\right\} .
\end{gathered}
$$

From Lemma 5 and Lemma 8, $\left\{u_{n}\right\}$ has a convergent subsequence (still denoted by $\left\{u_{n}\right\}$ ) and there exists $u_{* *} \in$ $H_{0}^{1}(\Omega)$ such that $u_{n} \rightarrow u_{* *}$ in $H_{0}^{1}(\Omega)$. Moreover, we can obtain $u_{* *}$ is a nonnegative weak solution of system (1) and

$$
I_{\lambda}\left(u_{* *}\right)=\lim _{n \rightarrow \infty} I_{\lambda}\left(u_{n}\right)=c>0 .
$$

Therefore, we infer that $u_{* *} \equiv 0$. Similar to Theorem 6 , we obtain $u_{* *}>0$ in $\Omega$, and so $\left(u_{* *}, \phi_{u_{* *}}\right)$ is a positive solution of system (1). The theorem is proved.

\section{Existence of a Ground State Solution of System (1)}

Define

$$
N=\inf \left\{I_{\lambda}(u): u \in H_{0}^{1}(\Omega), u \neq 0, I_{\lambda}^{\prime}\left(u_{n}\right)=0\right\}
$$

By the definition of $N$, there exists $\{u\} \in H_{0}^{1}(\Omega)$ such that $u_{n} \neq 0$, and

$$
I_{\lambda}\left(u_{n}\right) \rightarrow N, I_{\lambda}^{\prime}\left(u_{n}\right) \rightarrow 0 \text { as } n \rightarrow \infty \text {. }
$$

Obviously, we can easily deduce that $\left\{u_{n}\right\}$ is bounded in $H_{0}^{1}(\Omega)$. There then exist a nonnegative subsequence of $\left\{u_{n}\right\}$ $\left(I_{\lambda}\left(u_{n}\right)=I_{\lambda}\left(\left|u_{n}\right|\right)\right)$, still denoted by itself, and $u_{\lambda} \in H_{0}^{1}(\Omega)$ such that $u_{n} \rightarrow u_{\lambda}$ weakly in $H_{0}^{1}(\Omega)$. We claim that $u_{\lambda} \neq 0$. Arguing by contradiction, $u_{n} \rightarrow 0$ weakly in $H_{0}^{1}(\Omega)$ and $u_{n}$ $\rightarrow 0$ strongly in $L^{p}(\Omega)(1 \leq p<6)$. Then, we have

$$
\int_{\Omega} f_{\lambda}(x) u_{n}^{q} d x=o(1) .
$$

Consequently, from (53), it holds that

$$
\left\|u_{n}\right\|^{2}-\int_{\Omega}\left|u_{n}\right|^{6} d x=o(1) .
$$

Set $\lim _{n \rightarrow \infty}\left\|u_{n}\right\|=A$, note that $\int_{\Omega}\left|u_{n}\right|^{6} d x \leq S^{-3}\left\|u_{n}\right\|^{6}$, it follows

$$
A^{2} \geq S^{3 / 2}
$$

or

$$
A=0 \text {. }
$$

So it follows from (53) and (54) that

$$
\begin{aligned}
N= & \lim _{n \rightarrow \infty}\left\{\frac{1}{2}\left\|u_{*}\right\|^{2}-\frac{1}{4} \int_{\Omega} \phi_{u_{n}} u_{n}^{2} d x\right. \\
& \left.-\frac{1}{6} \int_{\Omega}\left|u_{n}\right|^{6} d x-\frac{1}{q} \int_{\Omega} f_{\lambda}(x) u_{n}^{q} d x\right\} \\
= & \lim _{n \rightarrow \infty} \frac{1}{4}\left\|u_{*}\right\|^{2}=\frac{1}{4} A^{2} .
\end{aligned}
$$

If $A=0$, then $N=0$, this contradicts to Theorem 6. Consequence, $N \geq(1 / 3) S^{3 / 2}$. According to Lemma 8 , we have ( 1 13) $S^{3 / 2} \leq N<(1 / 3) S^{3 / 2}-D \lambda^{2 /(2-q)}$, this is a contradiction. Consequently $u_{n} \rightarrow u_{\lambda} \neq 0$ in $H_{0}^{1}(\Omega)$. Moreover, by Lemma 5 , we can prove that $u_{n} \rightarrow u_{\lambda}$ in $H_{0}^{1}(\Omega)$, and $\left(u_{\lambda}, \phi_{u \lambda}\right)$ is a positive solution of system (1) with $I_{\lambda}\left(u_{n}\right) \geq N$. Next, we prove that $I_{\lambda}\left(u_{n}\right) \leq N$. Indeed, since $I_{\lambda}^{\prime}\left(u_{\lambda}\right)=0$, it follows

$$
\begin{aligned}
I_{\lambda}\left(u_{\lambda}\right)= & \frac{1}{2}\left\|u_{\lambda}\right\|^{2}-\frac{1}{4} \int_{\Omega} \phi_{u_{\lambda}} u_{\lambda}^{2} d x-\frac{1}{6} \int_{\Omega} u_{\lambda}^{6} d x \\
& -\frac{1}{q} \int_{\Omega} f_{\lambda}(x) u_{\lambda}^{q} d x-\frac{1}{4}\left\{\left\|u_{\lambda}\right\|^{2}-\int_{\Omega} \phi_{u_{\lambda}} u_{\lambda}^{2} d x\right. \\
& \left.-\int_{\Omega} u_{\lambda}^{6} d x-\int_{\Omega} f_{\lambda}(x) u_{\lambda}^{q} d x\right\} \\
= & \frac{1}{4}\left\|u_{\lambda}\right\|^{2}-\left[\frac{1}{q}-\frac{1}{4}\right] \int_{\Omega} f_{\lambda}(x) u_{\lambda}^{q} d x .
\end{aligned}
$$

In addition, it follows from (53) and the weak lower semicontinuity of the norm; it holds that

$$
\begin{aligned}
N= & \lim _{n \rightarrow \infty}\left[\frac{1}{2}\left\|u_{n}\right\|^{2}-\frac{1}{4} \int_{\Omega} \phi_{u_{n}} u_{n}^{2} d x-\frac{1}{6} \int_{\Omega}\left|u_{n}\right|^{6} d x\right. \\
& -\frac{1}{q} \int_{\Omega} f_{\lambda}(x) u_{n}^{q} d x-\frac{1}{4}\left\{\left\|u_{n}\right\|^{2}-\int_{\Omega} \phi_{u_{n}} u_{n}^{2} d x\right. \\
& \left.\left.-\int_{\Omega} u_{n}^{6} d x-\int_{\Omega} f_{\lambda}(x) u_{n}^{q} d x\right\}\right] \\
= & \lim _{n \rightarrow \infty}\left[\frac{1}{2}\left\|u_{\lambda}\right\|^{2}-\left(\frac{1}{q}-\frac{1}{4}\right) \int_{\Omega} f_{\lambda}(x) u_{\lambda}^{q} d x\right] \\
\geq & \frac{1}{4}\left\|u_{\lambda}\right\|^{2}-\left[\frac{1}{q}-\frac{1}{4}\right] \int_{\Omega} f_{\lambda}(x) u_{\lambda}^{q} d x .
\end{aligned}
$$

Consequently, $u_{\lambda} \neq 0$ and $I_{\lambda}\left(u_{\lambda}\right)=N$, which implies that $\left(u_{\lambda}, \phi_{u_{\lambda}}\right)$ is a positive ground state solution of system (1). Hence, we finish the proof of Theorem 1.

\section{Data Availability}

No data were used to support this study.

\section{Conflicts of Interest}

The authors declare that they have no conflicts of interest. 


\section{Acknowledgments}

This work was supported by the National Natural Science Foundation of China (No. 11661021).

\section{References}

[1] H. Liu, "Positive solutions of an asymptotically periodic Schrodinger-Poisson system with critical exponent," Nonlinear Analysis: Real World ApplicationsNonlinear Analysis: Real World Applications, vol. 32, pp. 198-212, 2016.

[2] J. Sun and S. Ma, "Ground state solutions for some Schrodinger-Poisson systems with periodic potentials," Journal of Differential Equations, vol. 260, no. 3, pp. 2119-2149, 2016.

[3] J. Zhang, "Ground state and multiple solutions for Schrödinger-Poisson equations with critical nonlinearity," Journal of Mathematical Analysis and Applications, vol. 440, no. 2, pp. 466-482, 2016.

[4] Z. Liu, Z. Zhang, and S. Huang, "Existence and nonexistence of positive solutions for a static Schrodinger- Poisson-Slater equation," Journal of Differential Equations, vol. 266, no. 9, pp. 5912-5941, 2019.

[5] S. Khoutir, "Infinitely many high energy radial solutions for a class of nonlinear Schrodinger-Poisson systems in R3," Applied Mathematics Letters, vol. 90, pp. 139-145, 2019.

[6] E. H. Lieb, "Existence and uniqueness of the minimizing solution of Choquard's nonlinear equation," Studies in Applied Mathematics, vol. 57, no. 2, pp. 93-105, 1977.

[7] Z. Liu and S. Guo, "On ground state solutions for the Schrödinger-Poisson equations with critical growth," Journal of Mathematical Analysis and Applications, vol. 412, no. 1, pp. 435-448, 2014.

[8] C. Y. Lei and J. F. Liao, "Multiple positive solutions for Schrödinger-Poisson system involving singularity and critical exponent," Mathematical Methods in the Applied Sciences, vol. 42, no. 7, pp. 2417-2430, 2019.

[9] E. G. Murcia and G. Siciliano, "Least energy radial signchanging solution for the Schrodinger-Poisson system in $\mathbb{R}^{3}$ under an asymptotically cubic nonlinearity," Journal of Mathematical Analysis and Applications, vol. 474, no. 1, pp. 544571, 2019.

[10] W. H. Xie, H. B. Chen, and H. X. Shi, "Multiplicity of positive solutions for Schrödinger-Poisson system with a critical nonlinearity in $\mathbb{R}^{3}$," Bulletin of the Malaysian Mathematical Sciences Society, vol. 42, pp. 2657-2680, 2019.

[11] J. Wang, L. Tian, J. Xu, and F. Zhang, "Existence of multiple positive solutions for Schrödinger-Poisson systems with critical growth," Zeitschrift für Angewandte Mathematik und Physik, vol. 66, no. 5, pp. 2441-2471, 2015.

[12] B. Almuaalemi, H. Chen, and S. Khoutir, "Existence of nontrivial solutions for Schrödinger-Poisson systems with critical exponent on bounded domains," Bulletin of the Malaysian Mathematical Sciences Society, vol. 42, no. 4, pp. 1675-1686, 2019.

[13] C. O. Alves and G. M. Figueiredo, "Existence of positive solution for a planar Schrödinger-Poisson system with exponential growth," Journal of Mathematical Physics, vol. 60, no. 1, article 011503, 2019.

[14] H. Fan, "Multiple positive solutions for a Schrödinger-Poisson involving convave-convex nonlinearities," Electronic Journal of Differential Equations, vol. 2019, no. 86, pp. 1-19, 2019.
[15] A. Azzollini and P. d'Avenia, "On a system involving a critically growing nonlinearity," Journal of Mathematical Analysis and Applications, vol. 387, no. 1, pp. 433-438, 2012.

[16] A. A. Pomponio, "Ground state solutions for the nonlinesr Schrödinger-Maxwell equations," Journal of Mathematical Analysis and Applications, vol. 345, pp. 90-108, 2008.

[17] A. Azzollini, P. d'Avenia, and G. Vaira, "Generalized Schrodinger-Newton system in dimension $N \geqslant 3$ : Critical case," Journal of Mathematical Analysis and Applications, vol. 449, no. 1, pp. 531-552, 2017.

[18] C. M. Chu and C. L. Tang, "Multiple results for critical quasilinear elliptic systems involving concave-convex nonlinearities and sign-changing weight functions," Bulletin of the Malaysian Mathematical Sciences Society, vol. 36, pp. 789-805, 2013.

[19] K. Teng, "Ground state solutions for the non-linear fractional Schrödinger-Poisson system," Applicable Analysis, vol. 98, no. 11, pp. 1959-1996, 2018.

[20] S. Chen and X. Tang, "Ground state solutions of SchrodingerPoisson systems with variable potential and convolution nonlinearity," Journal of Mathematical Analysis and Applications, vol. 473 , no. 1, pp. 87-111, 2019.

[21] Q. Zhang, "Existence of positive solution to KirchhoffSchrödinger-Poisson system with strong singular term," Journal of Mathematical Physics, vol. 60, no. 4, article 041504, 2019.

[22] W. Gan and S. Liu, "Multiple positive solutions of a class of Schrodinger-Poisson equation involving indefinite nonlinearity in R3," Applied Mathematics Letters, vol. 93, pp. 111-116, 2019.

[23] A. M. Batista and M. F. Furtado, "Positive and nodal solutions for a nonlinear Schrödinger-Poisson system with signchanging potentials," Nonlinear Analysis: Real World Applications., vol. 39, pp. 142-156, 2018.

[24] C.-Y. Lei and H. M. Suo, "Positive solutions for a SchrödingerPoisson system involving concave-convex nonlinearities," Computers \& Mathematcs with Applications, vol. 74, no. 6, pp. 1516-1524, 2017.

[25] C. Y. Lei and G. S. Liu, "Multiple Positive solutions for a Schrödinger-Newton system with sign-changing potential," Computers \& Mathematcs with Applications, vol. 77, no. 3, pp. 631-640, 2019.

[26] H. Brézis and E. Lieb, "A relation between pointwise convergence of functions and convergence of functionals," Proceedings of the American Mathematical Society, vol. 88, no. 3, pp. 486-490, 1983.

[27] H. Brézis and L. Nirenberg, "Positive solutions of nonlinear elliptic equations involving critical Sobolev exponents," Communications on Pure and Applied Mathematics, vol. 36, no. 4, pp. 437-477, 1983.

[28] A. Ambrosetti and P. H. Rabinowitz, "Dual variational methods in critical point theory and applications," Journal of Functional Analysis, vol. 14, no. 4, pp. 349-381, 1973. 